\title{
Reproductive alterations in hyperinsulinemic but normoandrogenic MSG obese female rats
}

\author{
Renato Simões Gaspar1, Renata Ohana Alves Benevides', \\ João Lucas de Lima Fontelles1, Caroline Castro Vale1, Lucas Martins França1, \\ Paulo de Tarso Silva Barros',2 and Antonio Marcus de Andrade Paes \\ 1Department of Physiological Sciences, Federal University of Maranhão, São Luís, Maranhão, Brazil \\ 2Department of Physiology, School of Medicine of Ribeirão Preto, University of São Paulo, Ribeirão \\ Preto, São Paulo, Brazil
}

\author{
Correspondence \\ should be addressed \\ to $A$ M de A Paes \\ Email \\ marcuspaes@ufma.br
}

\begin{abstract}
Obesity and metabolic syndrome are the common causes of reproductive and fertility disorders in women. In particular, polycystic ovary syndrome, which is clinically characterized by hyperandrogenism, oligo/anovulation, and polycystic ovarian morphology, has been increasingly associated with metabolic disorders. However, given the broad interplay between metabolic and reproductive functions, this remains a field of intense research. In this study, we investigated the effect of monosodium L-glutamate (MSG)-induced obesity on reproductive biology of female rats. Newborn female rats were subcutaneously injected with MSG ( $4 \mathrm{~g} / \mathrm{kg} / \mathrm{day})$ or equiosmolar saline (CTR) each 2 days up to postnatal day (pnd) 10. On pnd 60, estrous cycle was evaluated using vaginal smears twice a day for 15 days, which showed MSG rats to be oligocyclic. Thereafter, animals were killed on estrous phase for blood and tissue collection. MSG rats had increased body mass, accumulation of retroperitoneal and visceral fat pads, and visceral adipocyte hypertrophy compared with CTR rats. MSG rats were also dyslipidemic and hyperinsulinemic but were normoglycemic and normoandrogenic. Ovarian morphology analysis showed that MSG rats had a two-fold decrease in oocyte count but a six-fold increase on ovarian follicular cysts, along with a higher number of total primordial and atretic follicles. Moreover, MSG rats had a four-fold increase in anti-Müllerian hormone immunohistochemical staining on antral follicles. Taken together, data presented here characterize MSG obesity as a unique model to study the metabolic pathways underlying reproductive disorders in the absence of overactivated hypothalamic-pituitary-gonadal axis.
\end{abstract}

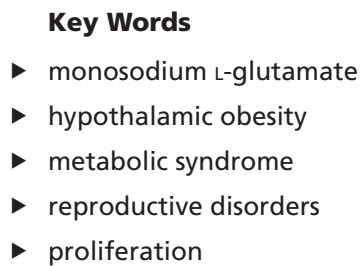

Journal of Endocrinology (2016) 229, 61-72

\section{Introduction}

Obesity and metabolic syndrome (MetS) have escalating prevalence worldwide and have reached an epidemic proportion, affecting the world population regardless of ethnicity or Human Development Index
(O’Neill \& O'Driscoll 2015). The latest consensus defines MetS as the presence of three of the five metabolic dysfunctions: obesity/central obesity, insulin resistance, dyslipidemia mainly as hypertriglyceridemia, hypertension,

Published by Bioscientifica Ltd. 
and fasting hyperglycemia (Alberti et al. 2009). Of the abovementioned dysfunctions, obesity is of paramount importance in the development of MetS as it seems to precede other MetS features ( $\mathrm{O}^{\prime}$ Neill \& O'Driscoll 2015). Concerning women's health, obesity and MetS are of special importance as epidemiological studies have shown that obesity is more prevalent in women (Wells et al. 2012), increasing their mortality risk (O'Neill \& O'Driscoll 2015) and predisposition for severe disturbances on reproductive physiology (Vryonidou et al. 2015).

The association between MetS risk factors, especially obesity and insulin resistance, and ovarian dysfunction has been reported in female mice (Wu et al. 2014b), rats (Sanchez-Garrido et al. 2015), and mini pigs (NewellFugate et al. 2014). Metabolic disturbances cause defects in the hypothalamic-pituitary-gonadal (HPG) axis via insulin and leptin feedback, which contributes to alter reproductive hormone levels (Watanobe 2002, Wu et al. 2014b). Obesity causes infertility in women, and even a modest 5\% loss in body weight can lead to ovulation (Duval et al. 2015). Furthermore, experimental models to study the effects of obesity on ovarian microenvironment and its reproductive outcomes often present hyperandrogenism, longer and irregular estrous cycle, and polycystic ovaries (Shi et al. 2009, NewellFugate et al. 2014, Wu et al. 2014b). Such characteristics are hallmarks of polycystic ovary syndrome (PCOS), the main reproductive disorder associated with obesity and MetS (Rotterdam 2004). Nevertheless, the characterization of novel approaches to investigate the interplay between MetS and female reproductive disturbances remains a field of intense research.

On this matter, the monosodium L-glutamate (MSG)-induced obesity model, first described by Olney (1969), shows unique MetS-related characteristics. MSG administration to newborn rodents results in damage to the arcuate nucleus and median eminence of hypothalamus (Olney 1969, Olney \& Sharpe 1969), decreasing growth hormone releasing hormone (GHRH) release, which impairs pulsatile growth hormone (GH) secretion by anterior pituitary somatosomes (Maiter et al. 1991). During adulthood, MSG rodents show stunted growth, mild obesity, increased fat accumulation, dyslipidemia, hyperleptinemia, hyperinsulinemia, and insulin resistance, which characterize it as a mild MetS model (Zhang et al. 2010, Franca et al. 2014, Miranda et al. 2014). Besides metabolic repercussions, MSG rodents show impaired reproductive parameters associated with dysfunctional HPG axis (Lamperti \& Baldwin 1983, Dada \& Blake 1985, Rose \& Weick 1987, Franca et al. 2006). Albeit not being sterile, as initially suggested (Olney 1969), MSG female rodents exhibit reduced ovarian weight (Lamperti \& Blaha 1976), longer estrous cycle (Nagasawa et al. 1974), delayed puberty (Lorden \& Caudle 1986), and reduced fertility (Campos et al. 2008). However, ovarian morphology of MSG rodents has deserved little attention in the literature. Sasaki and Sano (1986) reported increased ovarian follicular atresia with no corpora lutea in MSG mice, whereas Bojanić et al. (2009) reported many atretic follicles with unspecific ovarian degeneration in MSG rats. More recently, it was shown that 75-dayold MSG mice presented an increased number of primary ovarian follicles, with no further analysis (Das \& Ghosh 2011).

Notwithstanding the abovementioned features, there is scarce reports assessing the repercussions of metabolic and reproductive disturbances found in MSG animals on ovarian morphology. Therefore, in this study, we investigated the reproductive outcomes, particularly in the context of PCOS-like features, observed in MSG-induced obese female rats. To seek this purpose, morphometric, biochemical, and histological analyses were conducted in 75-day-old control and MSG female rats as an attempt to support the MSG obesity as a unique and affordable model to study how MetS may influence reproductive function in an animal without overstimulation of HPG axis.

\section{Materials and methods}

\section{Animals}

Pregnant Wistar rats were provided from the Animal Facility House of the Federal University of Maranhão, São Luís-MA, Brazil. After delivery, female newborns were used in this study. All animals were kept in a $12 \mathrm{~h}$ light: $12 \mathrm{~h}$ darkness cycle, controlled temperature $\left(22-24^{\circ} \mathrm{C}\right)$, with access to water and food ad libitum, in cages with up to four animals. Each group came from different mothers, with eight pups per litter. All procedures were performed in accordance with the National Council for the Control of Animal Experimentation (CONCEA) and approved by the Animal Care and Welfare Committee of the Federal University of Maranhão under ruling number 016/13. 


\section{Study procedure}

All newborn animals received subcutaneous injections of MSG ( $4 \mathrm{~g} / \mathrm{kg} /$ day; Sigma-Aldrich; MSG group, $n=7$ ) or equiosmolar saline (CTR group, $n=8$ ) on alternate days, from postnatal day (pnd) 2 through 10 (Olney 1969). Animals were weaned at pnd 21. On pnd 60, the Lee Index ((body weight ${ }^{1 / 3}(\mathrm{~g}) /$ naso-anal length $\left.\left.(\mathrm{cm})\right) \times 1000\right)$ (Bernardis \& Patterson 1968) was performed as a body mass index and vaginal smears were started to be collected. At pnd 73, animals were fasted for $8 \mathrm{~h}$ and tail capillary blood was collected to assess glycemia (strip test Accu-check Active; Roche Diagnostics; $10 \mathrm{mg} / \mathrm{dL}$ sensitivity and CV 2\%), triglyceridemia $(3 \mathrm{mg} / \mathrm{dL}$; CV 3.5\%), and cholesterolemia (1.04 mg/dL CV 2\%) (Labtest, Lagoa Nova, MG, Brazil). Oral glucose tolerance test (OGTT) was conducted in overnight fasted rats by administration of $4 \mathrm{~g} / \mathrm{kg}$ glucose and glycemia measurements $0,15,30,60$, and 120 min after gavage. Insulinemia was determined by radioimmunoassay with 125I (Genesis, São Paulo, SP, Brazil) as described previously (Ribeiro et al. 2015). Sensitivity was $0.1 \mathrm{ng} / \mathrm{mL}$ and intraassay CV was $0.075 \%$. To estimate insulin resistance, both the HOMA1-IR (Wallace et al. 2004) and the TyG (Simental-Mendia et al. 2008) indices were calculated. Rats were killed on estrous phase, between pnd 75 and pnd 80. Upon killing, the Lee Index was redone and animals were anesthetized with ketamine $(70 \mathrm{mg} / \mathrm{kg})$ and xylazine $(10 \mathrm{mg} / \mathrm{kg}$ ) (Syntec, Cotia, SP, Brazil). Blood was collected by aorta puncture, centrifuged, and serum stocked at $-20^{\circ} \mathrm{C}$ until analysis. Ovaries, uterus, and retroperitoneal and gonadal (visceral) fat pads were weighted. Oviducts were collected bilaterally for fresh ex vivo oocyte count as described elsewhere (Bernuci et al. 2008). Ovaries and attached gonadal fat pads were fixed in $4 \%$ paraformaldehyde for $24 \mathrm{~h}$, and kept on $70 \%$ $\mathrm{v} / \mathrm{v}$ ethanol for histological processing.

\section{Estrous cycle assessment}

Vaginal smears were taken twice a day for at least 15 consecutive days to determine the phases of the estrous cycle and cycles were deemed as regular, irregular, or prolonged estrous, as described previously (Marcondes et al. 2002, Connor et al. 2012). Briefly, regular cycles were typically 4-5 days long with characteristic transition between phases, irregular cycles presented discrepant phase transitioning and/or aberrant duration of phases, whereas cycles with two or more days of estrous were deemed prolonged estrous.

\section{Ovarian morphology}

The right ovary was embedded in paraffin and cut into $4 \mu \mathrm{m}$ sections. Six serial sections, $44 \mu \mathrm{m}$ apart each, were stained with hematoxylin-eosin (HE) and analyzed under light microscopy. Follicles containing an oocyte were classified according to Hirshfield and Midgley (1978): primordial follicles consisted of one layer, flattened cells surrounding an oocyte; primary follicles had one layer of cuboidal cells; secondary follicles had more than one layer of cuboidal granulosa cells; antral follicles had multiple layers of cuboidal granulosa cells and contained one or more antral spaces, cumulus oophorus, and theca layer may also have been evident. Healthy follicles had no deformation on any cell layers or pyknotic nuclei. Atretic follicles were characterized by shrinkage or collapsing, lacking the oocyte or presenting granulosa cells with at least two pyknotic nuclei (Brawer et al. 1986, Wright et al. 1999, Lara et al. 2000, Webber et al. 2003). Cystic follicles were considered large fluid-filled cysts with diminished granulosa cell layer (up to four layers) and thickened theca interna cell layer (Brawer et al. 1986, Lara et al. 2000, Manneras et al. 2007, Shi et al. 2009). Micrographs were taken at $40 \times$ magnification.

\section{Immunohistochemistry}

For immunohistochemical staining, 3-aminopropyltriethoxysilane (Sigma-Aldrich) slides were used. Sections were deparaffinized, blocked for endogenous peroxidase activity, washed with PBS, and immersed in $90^{\circ} \mathrm{C}$ citrate buffer. Slides were then blocked for non-specific binding in $5 \% \mathrm{v} / \mathrm{v}$ albumin-phosphate buffer. Primary antibody for anti-Müllerian hormone (AMH) (R\&D Systems) was incubated at 1:400 dilutions at $4^{\circ} \mathrm{C}$ overnight. Slides were rinsed in PBS and incubated for $2 \mathrm{~h}$ at room temperature with secondary anti-goat antibody (R\&D Systems) and then with streptavidin-biotin peroxidase complex (avidin biotin complex; Vector Laboratories, Burlington, ON, Canada). The peroxidase activity was developed with 3,3-diaminobenzidine tetrahydrochloride (SigmaAldrich) in distilled water and $0.03 \% \mathrm{v} / \mathrm{v}_{2} \mathrm{O}_{2}$. After a final PBS wash, all sections were counterstained with hematoxylin. A negative control of primary antibody immunostaining is shown in Supplementary Fig. 1 (see section on supplementary data given at the end of this article). Three serial sections per animal were stained and analyzed under light microscopy. Follicles were divided into preantral (primordial, primary, and 
Table 1 Morphometric parameters of CTR and MSG obese rats.

\begin{tabular}{l} 
\\
\hline Morphometric parameters \\
Body weight $(\mathrm{g})$ \\
Naso-anal length $(\mathrm{cm})$ \\
Lee Index $\left(\left(\mathrm{g}^{1 / 3} / \mathrm{cm}\right) \times 1000\right)$ \\
Retroperitoneal fat pad \\
$(\mathrm{g} / 100 \mathrm{~g} \mathrm{BW})$ \\
Visceral fat pad \\
$(\mathrm{g} / 100 \mathrm{~g} \mathrm{BW})$ \\
Uterus $(\mathrm{g} / 100 \mathrm{~g} \mathrm{BW})$ \\
Ovaries $(\mathrm{mg} / 100 \mathrm{~g} \mathrm{BW})$ \\
\hline
\end{tabular}

$\begin{array}{r}\hline \multicolumn{1}{c}{\text { CTR }} \\ \hline 166.40 \pm 3.45 \\ 17.70 \pm 0.12 \\ 310.60 \pm 1.71 \\ 1.08 \pm 0.17 \\ 1.08 \pm 0.18 \\ 0.34 \pm 0.02 \\ 0.15 \pm 0.01 \\ \hline\end{array}$

$\begin{array}{r}\hline \text { MSG } \\ 158.00 \pm 3.70 \\ 16.03 \pm 0.22 * * \\ 337.30 \pm 2.99 * * \\ 2.84 \pm 0.26 * * \\ 3.67 \pm 0.33 * * \\ 0.20 \pm 0.02 * * \\ 0.07 \pm 0.00 * * \\ \hline\end{array}$

Results are mean \pm S.E.M. of $n=5-7 .{ }^{*} * P<0.001$ vs CTR.

secondary) and antral. Micrographs were taken at 100x and 200x magnifications. Percentage of follicle area stained with AMH was calculated using ImageJ software $1.48 \mathrm{v}$ (National Institutes of Health, Bethesda, MD, USA; http://imagej.nih.gov.ij).

\section{Measurement of adipocyte area}

Gonadal fat pads were processed together with the right ovary and cut into $4 \mu \mathrm{m}$ sections. One hundred adipocytes were analyzed from each animal. All sections were at least $100 \mu \mathrm{m}$ apart to prevent multiple counts of the same adipocyte. Micrographs were taken at 200× magnification. The adipocyte area was calculated using AxionVision (AxioVs40x64 V 4.9.1.0; Carl Zeiss Microscopy GmbH).
Subsequently, the frequency of adipocytes per similar size class with intervals of $500 \mu^{2}$ was calculated.

\section{Serum reproductive hormones}

Steroidal hormones were extracted from serum before the assay. Briefly, serum was thawed on ice; diethyl ether $5 \mathrm{v} / \mathrm{v}$ was added and thoroughly mixed. This mixture was centrifuged under $1000 \boldsymbol{g}$ for $3 \mathrm{~min}$ to separate phases. Upper layer (ether) was then stocked on a clean tube and was repeated twice for optimal extraction. A precision curve was run for a coefficient value of $r^{2}>0.96$. Estradiol and testosterone assays (Roche Diagnostics $\mathrm{GmbH}$ ) were performed on the same day. Sensitivity was $5 \mathrm{pg} / \mathrm{mL}$ and $0.25 \mathrm{ng} / \mathrm{mL}$ respectively. $\mathrm{CV}$ was $2.73 \%$ and $<1 \%$ respectively. Luteinizing hormone (LH) serum levels were not extracted before the assay and were measured by enzyme-linked immunosorbent assay (ELISA) kit as per manufacturer's instructions (Elabscience Biotechnology Co. Ltd, Wuhan, China). Sensitivity was $0.938 \mathrm{mIU} / \mathrm{mL}$ and $\mathrm{CV}<10 \%$.

\section{Statistical analyses}

The results are expressed as mean \pm s.E.M. on the tables and bar graphs. On the box plot graphs, the box shows the 25th and 75th percentiles and the middle line is considered the median. The whiskers indicate the upper and lower values not classified as statistical outliers. Student's $t$-test was used for comparison and differences were considered significant at $P<0.05$.
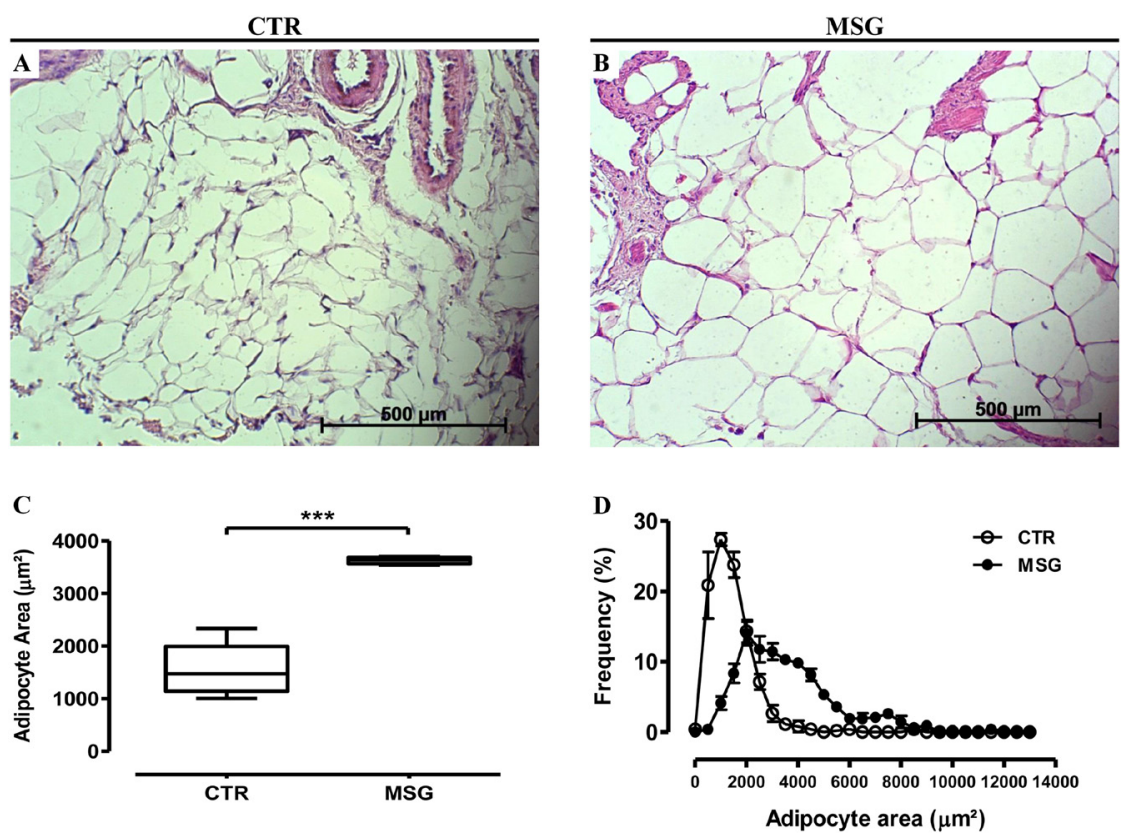

Figure 1

Increased periovarian adipocyte area in MSG obese rats. ( $A$ and $B$ ) Representative sections of paraffin-embedded and hematoxylin-eosinstained periovarian fat pads from CTR $(A, n=5)$ and MSG (B, $n=4)$ animals. (C) Mean adipocyte area demonstrated by box and whiskers plot illustrating median (central line), range (whiskers), and 25th and 75th percentiles (box) of at least 97 adipocytes per animal. (D) Adipocyte area distribution of CTR (open circles) and MSG (black circles) rats. ${ }^{*} * P<0.001$ compared with CTR by Student's $t$-test. A full colour version of this figure is available at http://dx.doi. org/10.1530/JOE-15-0453. 
A

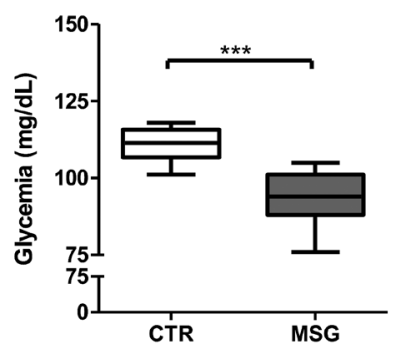

E

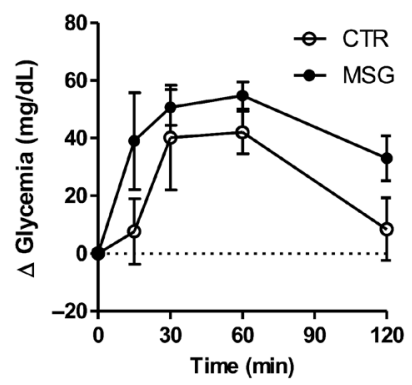

B

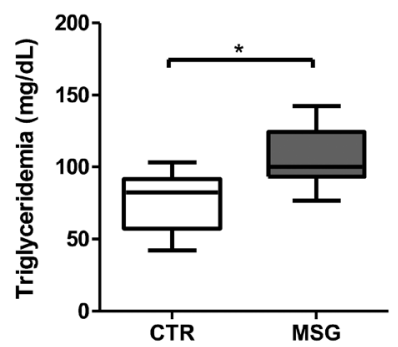

F

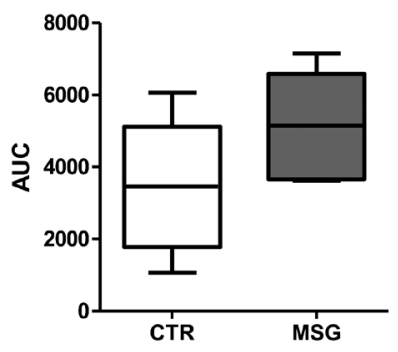

C

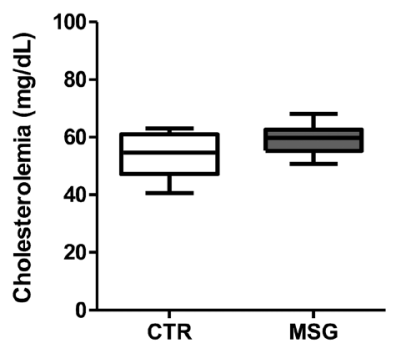

G

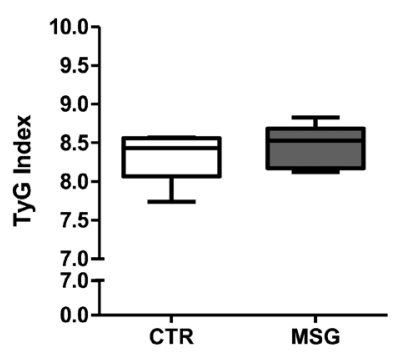

D

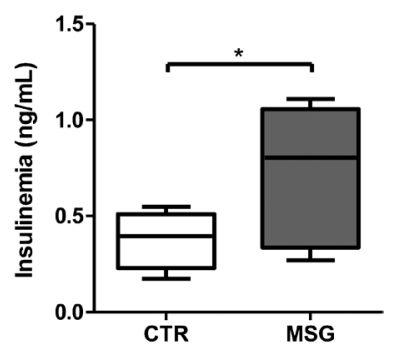

H

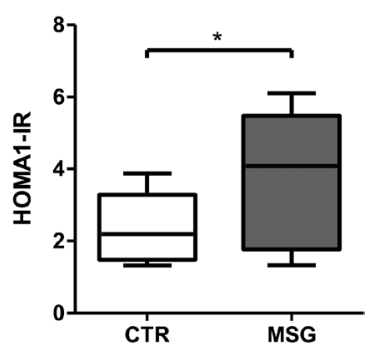

Figure 2

Serum metabolic profile of CTR and MSG animals. Fasted glycemia (A) measured by strip test. Fasted triglyceridemia (B) and cholesterolemia (C) determined by spectrophotometric assay kits. Fasted insulinemia (D) measured by radioimmunoassay. (E) Oral glucose tolerance test (OGTT) conducted in fasting CTR and MSG animals. (F) Area under the curve (AUC) of OGTT expressed no difference between groups. (G) TyG Index was calculated based on fasted glycemia and triglyceridemia: In (fasting glycemia $(\mathrm{mg} / \mathrm{dL}) \times$ fasting triglyceridemia $(\mathrm{mg} / \mathrm{dL}) / 2)$. (H) HOMA1-IR index calculated based on fasted glycemia and insulinemia: (fasting insulinemia $(\mu \mathrm{U} / \mathrm{mL}) \times$ fasting glycemia $(\mathrm{mmol} / \mathrm{L}) / 22.5)$. All data expressed as box and whiskers plot: median (central line), range (whiskers), and 25 th and 75th percentiles (box) with $n=6-8$. * $P<0.05$ vs CTR; *** $P<0.001$ vs CTR analyzed by Student's $t$-test.

\section{Results}

\section{Morphometric parameters}

Morphometric parameters were measured on pnd 75 for both CTR and MSG female rats (Table 1). Body weight did not differ between groups; however, MSG animals were shorter than CTR animals, which contributed to a higher body mass in MSG, as inferred from Lee Index calculation. Moreover, MSG rats presented higher white adipose tissue accumulation. Specifically, retroperitoneal and visceral fat pads were increased by almost 3- and 3.5fold, respectively, compared with CTR rats. Interestingly, both uterus and ovaries were lighter in MSG rats, probably due to decreased GH serum levels, a hallmark of this model.

Panels A and B in Fig. 1 show visceral fat attached to ovaries stained with HE for CTR and MSG respectively. The mean adipocyte area from each animal was two-fold increased in MSG animals compared with CTR animals (CTR $1551 \pm 223.70$ vs MSG $3632 \pm 32.73 \mu \mathrm{m}^{2}, P<0.001$; Fig. 1C). Noteworthy, the histogram of adipocyte area distribution indicated that MSG animals had increased frequency of large adipocytes, shifting the curve to the right (Fig. 1D). These findings reiterate an imbalance on the metabolic aspects of MSG rats, which could impair reproductive functions on these animals.

\section{Serum metabolic and reproductive hormone profiles}

Assessment of serum metabolic parameters, as shown in Fig. 2, revealed that MSG rats had lower glycemia (CTR $111.00 \pm 1.98$ vs MSG 92.86 $\pm 3.59 \mathrm{mg} / \mathrm{dL}, P<0.01$; Fig. 2A) despite higher triglyceridemia (CTR 75.53 \pm 8.07 vs MSG $106.80 \pm 8.17 \mathrm{mg} / \mathrm{dL}, \quad P<0.05$; Fig. $2 \mathrm{~B})$, with no difference on cholesterolemia (CTR $53.11 \pm 3.06$ vs MSG 59.30 $\pm 2.04 \mathrm{mg} / \mathrm{dL}$; Fig. 2C). MSG animals were hyperinsulinemic, with roughly three times more fasting serum insulin levels than CTR animals (CTR 0.32 0.07 vs MSG $0.89 \pm 0.2 \mathrm{ng} / \mathrm{mL}, P<0.05$; Fig. 2D). OGTT was performed on fasting animals without significant difference between groups (Fig. 2E; CTR 3451 \pm 836.60 vs MSG 5191 $\pm 586.30 ;$ Fig. 2F). Insulin resistance was estimated by calculating both TyG (CTR $8.28 \pm 0.12$ vs MSG 8.48 0.10; Fig. 2G) and HOMA1-IR (CTR 2.06 \pm 0.44 vs MSG 4.56 $\pm 0.96, P<0.05$; Fig. $2 \mathrm{H})$ indices, which showed statistical difference only on the HOMA1-IR.

Published by Bioscientifica Ltd 
A

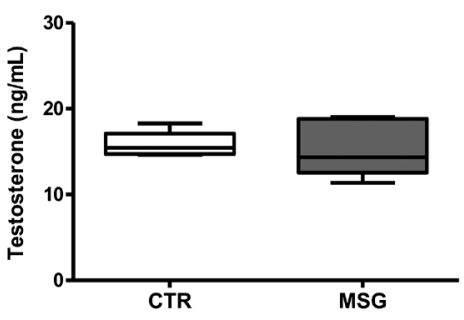

B

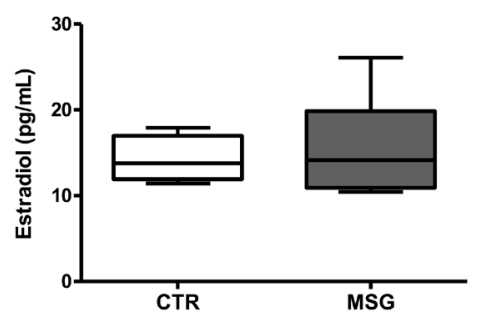

C

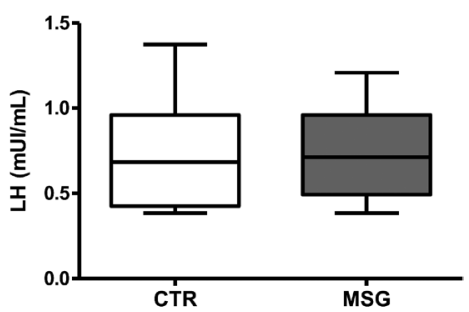

Figure 3

Serum reproductive hormones of CTR and MSG rats do not differ. All animals were on estrous upon blood collection. Serum testosterone (A) and estradiol (B) were extracted before the assay as described in the "Materials and methods" section. (C) Luteinizing hormone (LH) was measured without prior extraction. Data expressed as box and whiskers plot illustrating median (central line), range (whiskers), and 25th and 75th percentiles (box) with $n=4-7$. Student's $t$-test analysis showed no difference between groups.

Serum reproductive hormones of both groups are shown in Fig. 3. Surprisingly, no difference was found on serum testosterone (CTR $15.82 \pm 0.66$ vs MSG $15.15 \pm 1.30 \mathrm{ng} / \mathrm{mL} ;$ Fig. $3 \mathrm{~A})$, estradiol (CTR $14.23 \pm 1.36 \mathrm{vs}$ MSG $15.63 \pm 2.46 \mathrm{pg} / \mathrm{mL}$; Fig. 3B), or LH (CTR $14.23 \pm 1.36 \mathrm{vs}$ $15.63 \pm 2.46 \mathrm{MSG} \mathrm{mIU} / \mathrm{mL}$; Fig. 3C) levels. In spite of the absence of reproductive hormone alterations, our data support MSG female rats suffer from MetS, which might affect other ovarian functions.

\section{Cyclicity, ovulation and ovarian morphology}

Typically, CTR animals showed regular 4- to 5-day cycles, whereas MSG animals were oligocyclic, with less

A
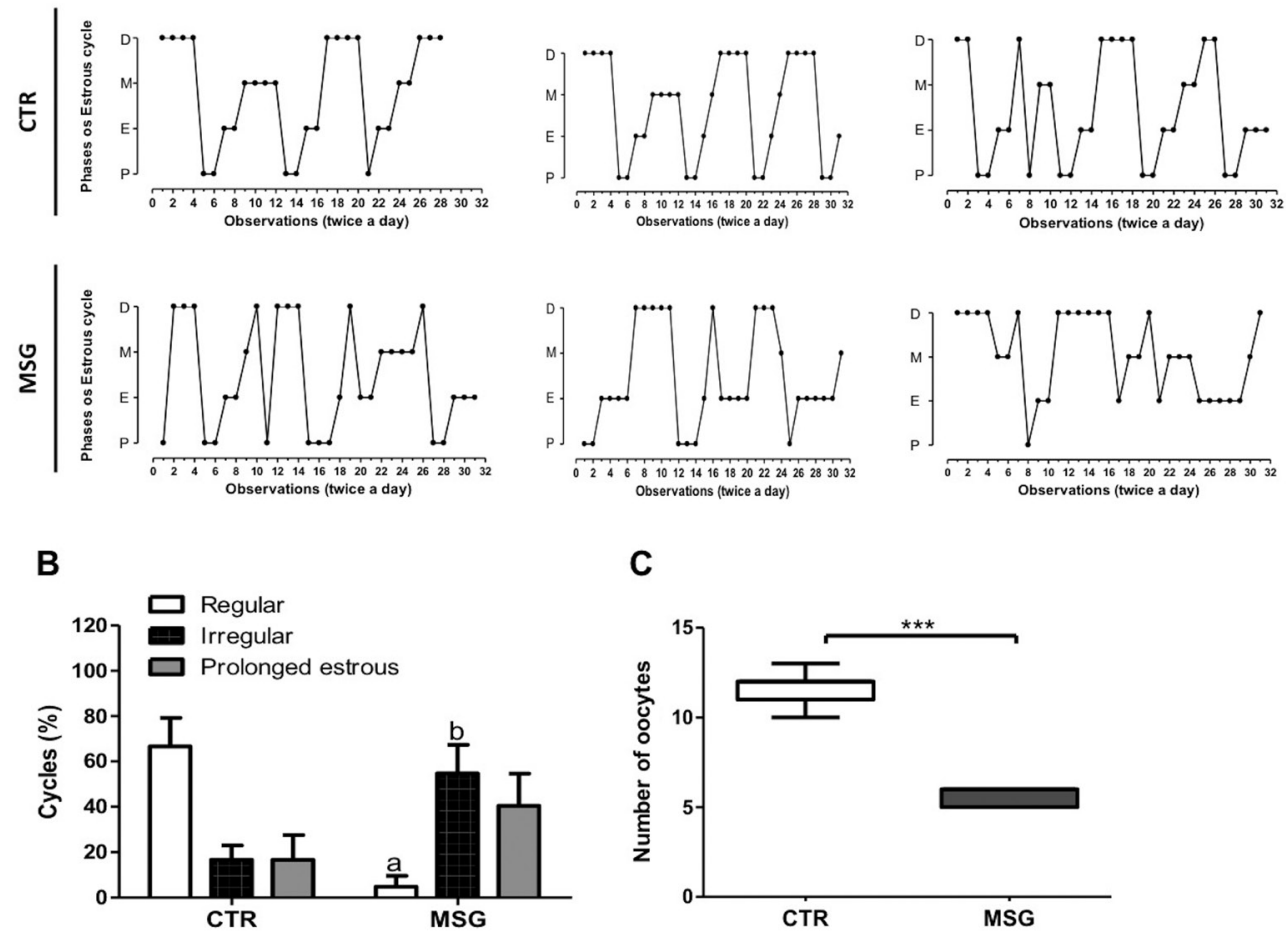

C

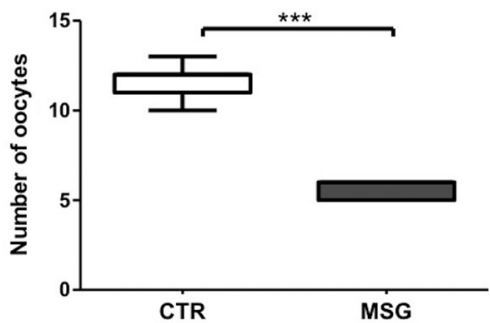

\section{Figure 4}

Hyperinsulinemic MSG animals exhibit irregular cycles and less ovulated oocytes. (A) Representative estrous cycles from three different CTR (top) and MSG (bottom) females taken twice a day from 60 to 75 pnd; P, proestrus; E, estrus; M, metaestrus; D, diestrus. (B) Estrous cycle classified as regular, irregular, or prolonged estrous according to the criteria exposed in the "Materials and methods" section; data presented as mean \pm s.E.M. (C) Mean fresh ex vivo oocyte count after ovulation on fallopian tubes from CTR and MSG rats demonstrated by box and whiskers plot illustrating median (central line), range (whiskers), and 25th and 75th percentiles (box). a $P<0.05$ vs regular CTR; $\mathrm{b} P<0.05$ vs irregular CTR; $* * * P<0.001$ vs CTR. All comparisons were analyzed by Student's $t$-test. $n=6-8$ per group. 

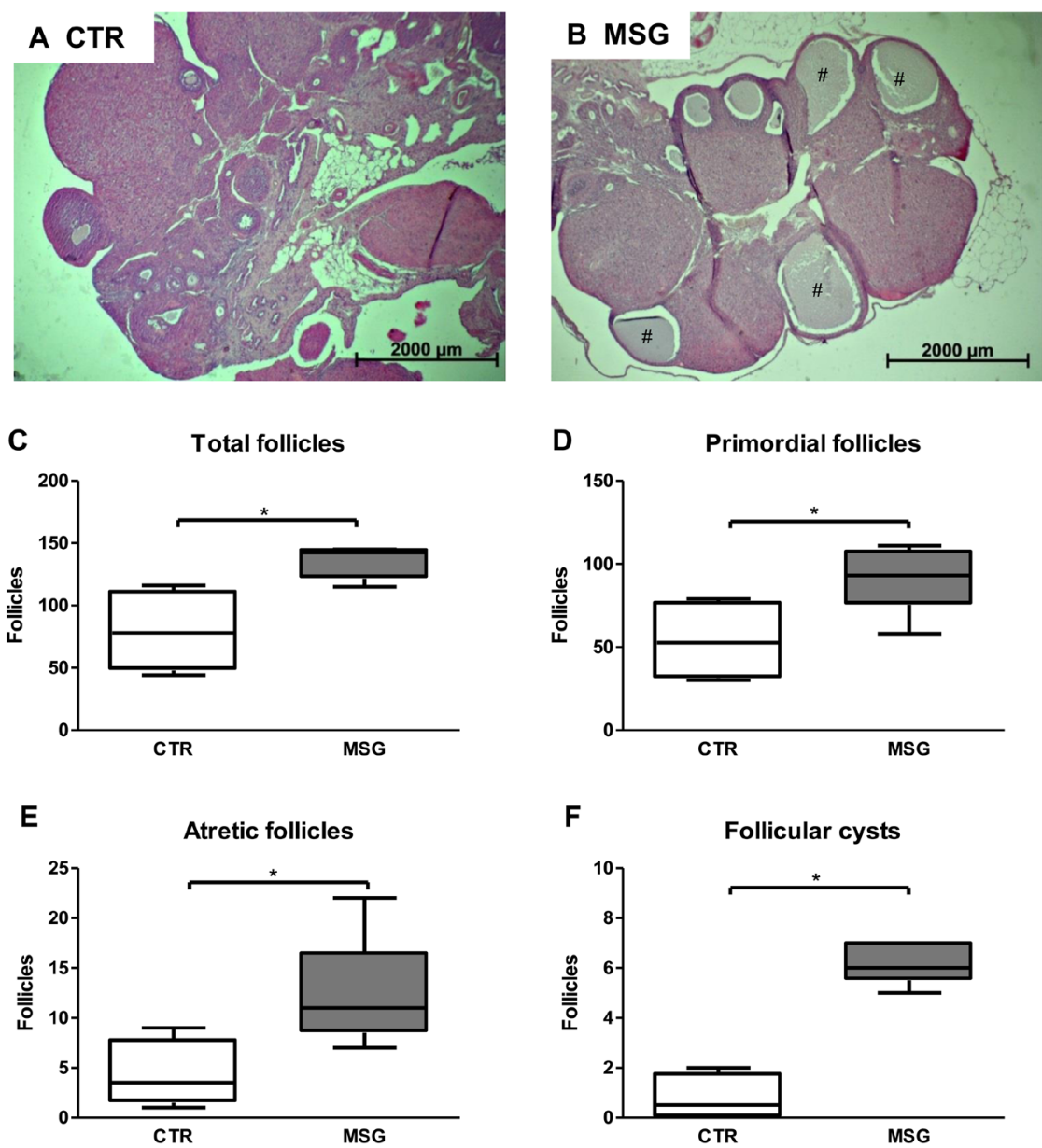

\begin{abstract}
Figure 5
Quantitative histological analysis of ovarian alterations in MSG obese rats. (A and B) Representative sections of paraffin-embedded and hematoxylin-eosin-stained ovaries from CTR (A) and MSG (B) rats; \# in B indicates follicular cysts. (C, D, E and F) Ovaries from CTR $(n=4)$ and MSG $(n=5)$ rats were embedded with paraffin and cut into $4 \mu \mathrm{m}$ sections. Every 11th section was stained with hematoxylin and eosin and analyzed for differential follicular counting according to the morphological pattern showed in each graph. Total follicles from CTR and MSG rats are demonstrated by box and whiskers plot illustrating median (central line), range (whiskers), and 25 and 75th percentiles (box). * $P<0.05$ vs CTR analyzed by Student's $t$-test. A full colour version of this figure is available at http://dx.doi.org/10.1530/JOE-15-0453.
\end{abstract}

proestrus-to-estrus progression and longer metaestrusdiestrus phase (Fig. 4A). This was statistically translated by decreased regular (CTR $66.67 \pm 12.60$ vs $4.76 \pm 4.76 \%$ MSG, $P<0.05$; Fig. 4B) and increased irregular (CTR 16.67 \pm 6.30 vs $54.76 \pm 12.58 \%$ MSG, $P<0.05$; Fig. $4 \mathrm{~B}$ ) cycles in MSG rats. There was no difference on prolonged estrous between groups (CTR $16.67 \pm 10.91$ vs $40.48 \pm 14.14 \%$ MSG, $P=0.19$; Fig. 4B). Ovulation was evaluated by fresh ex vivo oocyte count. Our MSG rats showed a twofold decrease in oocyte count compared with CTR (CTR $11.57 \pm 0.37$ vs MSG 5.57 $\pm 0.20, P<0.001$; Fig. $4 C)$. These results suggest that MSG rats have compromised fertility and reproductive function.

Ovary sections were then analyzed under light microscopy (Fig. 5). Representative sections from CTR (Fig. 5A) and MSG (Fig. 5B) rats displayed striking differences between groups regarding follicular cyst count. Figure 5C shows increased total follicle count in MSG animals (CTR 79.00 \pm 16.44 vs MSG 136.30 $\pm 7.13, P<0.05$; Fig. 5C), greatly due to increased primordial follicle

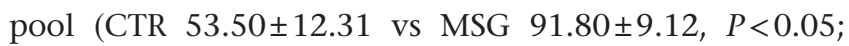

Fig. 5D). MSG animals also had a three-fold increase in atretic follicle number when compared with CTR animals (CTR $4.25 \pm 1.70$ vs MSG 12.20 $\pm 2.56, P<0.05$; Fig. 5E). Moreover, MSG animals had an astonishing six-fold increase on follicular cysts (CTR $0.75 \pm 0.48$ vs MSG 6.20 $\pm 0.37, P<0.01$; Fig. 5F). Taken together, these reproductive data support PCOS-like characteristics in MSG animals.

\section{AMH immunohistochemistry}

As $\mathrm{AMH}$ hypersecretion by granulosa cells is involved in follicle maturation, ovarian immunohistochemistry was performed. Data in Fig. 6A, B, C and D show higher intensity of $\mathrm{AMH}$ staining on MSG antral follicles compared with CTR. The quantitative analysis showed no difference in the percentage of staining among preantral follicles (Fig. 6E). However, there was a four-fold increase in AMH staining on antral follicles of MSG rats (CTR $2.07 \pm 0.59$ vs MSG 9.43 $\pm 2.06, P<0.01$; Fig. 6 F). Despite this, there was no significant difference in total follicle 

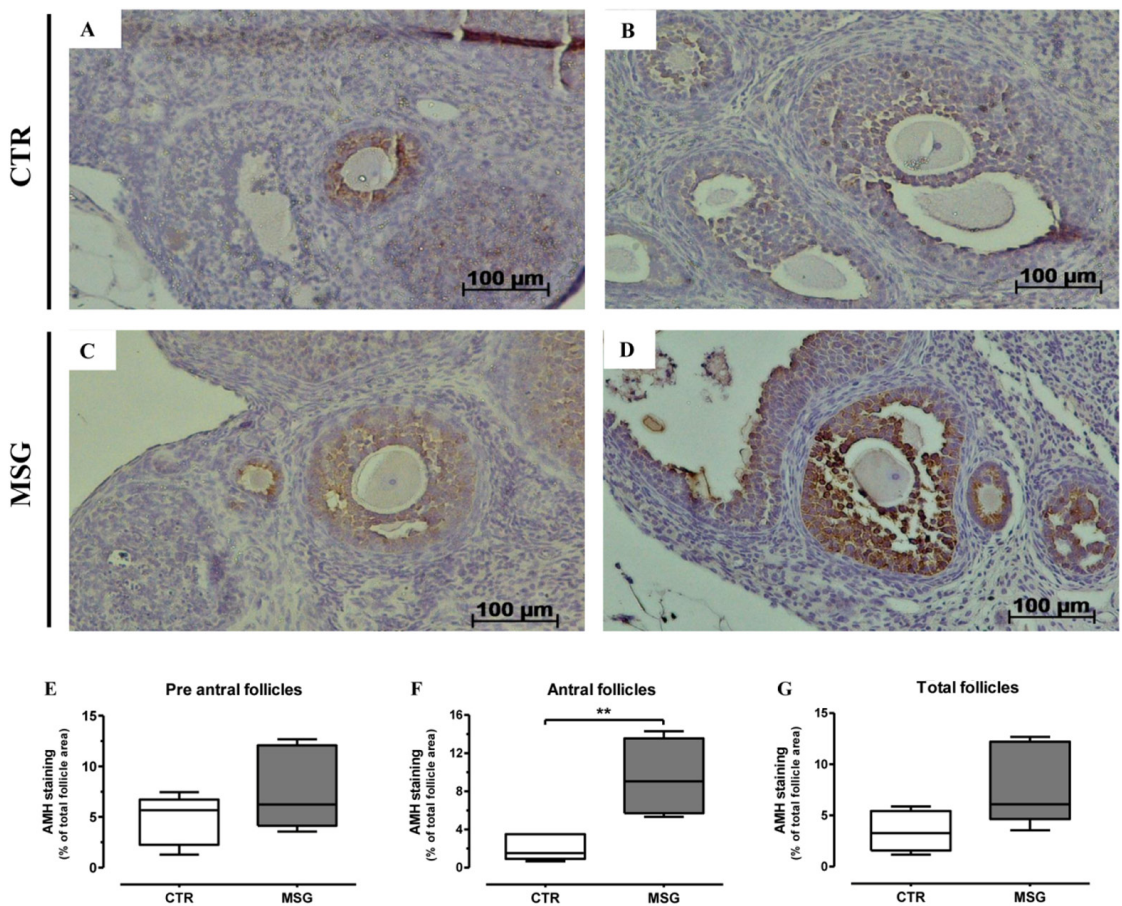

\section{Figure 6}

Immunohistochemical localization of antiMüllerian hormone (AMH) in the ovaries of CTR and MSG obese rats. Ovarian sections from CTR $(n=5)$ and MSG $(n=4)$ rats were stained with $\mathrm{AMH}$ and analyzed three serial sections per animal as described in the "Materials and methods" section. (A, B, C and D) Representative sections of $A M H$ staining on preantral and antral follicles are shown for CTR ( $A$ and $B$ ) and MSG ( $C$ and $D$ ) rats respectively. ( $E, F$ and $G$ ) Percentage of AMH staining on preantral (E), antral (F), and total (G) follicles area; mean percentage of staining in the ovaries from CTR and MSG rats are demonstrated by box and whiskers plot illustrating median (central line), range (whiskers), and 25 and 75 th percentiles (box). ${ }^{*} P<0.01$ vs CTR by Student's $t$-test. A full colour version of this figure is available at http:// dx.doi.org/10.1530/JOE-15-0453. staining (Fig. 6G). Altogether, these data are suggestive of a dysregulation on the granulosa cell layer with disrupted maturation of MSG ovarian follicles.

\section{Discussion}

This study strengthens the pivotal role of hyperinsulinemia and MetS-associated comorbidities in reproductive dysfunctions, resembling those present in PCOS. Subcutaneous injections of MSG to newborn female rats lead to hyperinsulinemia, although with unaltered serum testosterone, estradiol, or LH levels. MSG rats exhibited obesity, hypertriglyceridemia, and visceral adipocyte hypertrophy. Additionally, they had irregular estrous cycles, with oligo-ovulation, and increased number of ovarian follicular cysts and atretic follicles. Moreover, our MSG rats presented augmented AMH immunostaining on antral follicles and increased primordial follicle pool. Such characteristics suggest that MetS features, such as hyperinsulinemia, obesity, and adipocyte dysfunction, trigger reproductive deficits producing a PCOS-like phenotype despite the absence of hyperandrogenemia or increased LH levels. To the best of our knowledge, this report is the first to describe a MetS female model without overactivated HPG and one of the few to describe PCOS characteristics despite normoandrogenism. In tandem, our female MSG rat reveals itself to be an affordable model to study how MetS influences the reproductive system under no interference of increased serum HPG hormones.

Metabolic disturbances are highly prevalent among women diagnosed with PCOS and are considered to play an important role in the pathophysiology of this syndrome. Indeed, $50-80 \%$ of PCOS women are obese, whereas $44-70 \%$ are resistant to insulin (Dumesic et al. 2015). Obesity has been associated with more severe PCOS symptoms, whereas treatments directed to weight loss, such as exercise and anorexic drugs, significantly improve reproductive parameters. Numerous mechanisms correlating obesity and PCOS have been proposed, one of which consists in elevated insulinemia due to increased fat deposition, hypertriglyceridemia, and insulin resistance. It is important to notice that hyperinsulinemia has been proposed as a culprit for PCOS, and the concurrence of hyperinsulinemia/insulin resistance has higher prevalence in PCOS women, independent of body weight (Wu et al. 2014a, Dumesic et al. 2015).

Data presented herein show that our MSG rats developed MetS, translated by a rise in their Lee Index, accumulation of visceral and retroperitoneal fat pads, hypertriglyceridemia, and hyperinsulinemia. However, we found decreased serum glucose levels and unchanged OGTT in MSG rats. Data on insulin resistance were controversial, as TyG Index suggested that MSG rats have regular insulin sensitivity, whereas HOMA1-IR indicated the opposite. This could be explained by an exacerbated vagus nerve activity, a hallmark for MSG rodents, which

Published by Bioscientifica Ltd 
causes insulin oversecretion independently from serum glucose levels and insulin resistance (Miranda et al. 2014). In fact, even 6-month male MSG rats showed lower glycemia than control (Liu et al. 2011), yet there is scarce data concerning glycemia measurements on MSG females. Therefore, despite increased HOMA1-IR index, 75-day-old MSG females are likely to have preserved insulin sensitivity, which needs to be addressed through molecular methods in future studies.

Another important metabolic finding of our MSG rats was adipocyte hypertrophy on visceral periovarian fat pads. The mean adipocyte area of MSG animals was twofold higher than that of CTR animals, reflected by a rightsided shift in the histogram of adipocytes' area. Adipocyte enlargement is related to hyperinsulinemia (Gao et al. 2015) and hyperleptinemia (Couillard et al. 2000). Leptin affects the reproductive physiology evoking gonadotropinreleasing hormone $(\mathrm{GnRH})$ and $\mathrm{LH}$ secretion in rats (Watanobe 2002), while disturbing follicle maturation and ovulation (Escobar-Morreale \& San Millan 2007). Of interest, hyperleptinemia has been described in MSG female rats as young as 30 days old (Franca et al. 2006), reaching a ten-fold increase in 120-day-old female rats (Moreno et al. 2006). Thus, adipocyte hypertrophy showed herein might be related to increased leptin levels, which contribute to a disruption of female reproductive function. In humans, it has been shown that PCOS women have a $25 \%$ increase in adipocyte diameter when compared with BMI-matched non-PCOS subjects (Manneras-Holm et al. 2011), advocating for a relationship between PCOS and adipocyte hypertrophy.

Assessment of reproductive hormone levels showed that testosterone, estradiol, and LH were unaltered in our MSG rats. Neonatal MSG administration causes destruction of neuronal bodies in arcuate nucleus and median eminence of hypothalamus, disrupting the hypothalamic-pituitary axis (Lamperti \& Blaha 1976). However, it has been shown that axons and dendrites in passage through these nuclei are spared, maintaining a lower but preserved LH secretion (Dada \& Blake 1985). Moreover, facing an insufficient GnRH release, LH levels are maintained by input from anterior hypothalamic nucleus (Lamperti \& Baldwin 1983), as well as reorganization of other hypothalamic nuclei (Rose \& Weick 1987). Subsequently, MSG rodents have been shown to exhibit low to normal levels of reproductive hormones (Nemeroff et al. 1981, Lamperti \& Baldwin 1983, Dada \& Blake 1985, Franca et al. 2006), despite their gonadal atrophy (Table 1) and possible peripheral aromatization of androgens by white adipose tissue, as shown in other animal obese models (Newell-Fugate et al. 2014).
It has been reported that $\mathrm{LH}$ is the main inductor of ovarian androgen production (Knudsen et al. 2010), whereas hyperinsulinemia contributes to roughly $20 \%$ of serum testosterone levels (Azziz et al. 2001, Norman et al. 2002, Creanga et al. 2008). In humans (Tosi et al. 2012) and animal MetS models (Newell-Fugate et al. 2014, Wu et al. 2014b), hyperinsulinemia is able to cause elevation of LH secretion with consequent hyperandrogenemia, as hypothalamic nuclei structures are intact. However, in our MSG female rats, hyperinsulinemia seems to be unable to increase LH secretion, given the hypothalamic damage caused by MSG injection, resulting in lower to normal testosterone production, as shown in Fig. 3. Therefore, considering the normoandrogenemia verified in our MSG rats and their dysfunctional HPG axis, reproductive disorders described herein might be directly consequent to metabolic disturbances, i.e., hyperinsulinemia, obesity, and adipocyte dysfunction.

Our MSG rats exhibited irregular estrous cycles, which reinforces previous findings (Nagasawa et al. 1974, Lamperti \& Blaha 1976, Lorden \& Caudle 1986, Campos et al. 2008) and supports the connection between MetS and reproductive disorders. The MSG rats had lighter ovaries when compared with CTR, explained by deficient GH pulsatile secretion. They were oligocyclic rather than acyclic, with a higher frequency of irregular cycles and two-fold less oocyte on their fallopian tubes after ovulation. Of note, one could hypothesize the oligocycles/oligo-ovulation in MSG animals to be due to increased prolactin levels; however, it is well documented that MSG rodents, especially females, exhibit unchanged prolactinemia (Clemens et al. 1978, Nemeroff et al. 1981, Franca et al. 2006). Nonetheless, we recognize this as a limitation in this report and suggest future studies to investigate the effect of MSG obesity on the prolactin feedback loop. Noteworthy, our MSG obese rats present disrupted estrous cycle and oligo-ovulation patterns similar to female obese mice ( $\mathrm{Wu}$ et al. 2014b), rats (Manneras et al. 2007), and mini pigs (Newell-Fugate et al. 2014) with otherwise preserved HPG axis function, suggesting that the latter has a minor effect on MetS outcomes on estrous cycle and ovulation.

MetS and its highly associated reproductive disorder, PCOS, also cause anomalies on ovarian structures. For instance, PCOS women showed increased follicle count, primordial follicles, follicular cysts, and atresia (Rotterdam 2004, Shi et al. 2009, Walters et al. 2012). MetS female models, such as the genetically modified JCR:LA-cp rats, which has a malfunction on its leptin receptors, are also presented with PCO and follicle atresia (Shi et al. 2009),

Published by Bioscientifica Ltd 
whereas the New Zealand obese mice showed increased primordial follicle counts and follicle atresia without PCO (Radavelli-Bagatini et al. 2011). Diet-induced obese mini pigs were also reported to have cystic ovarian follicles (Newell-Fugate et al. 2014). Our MSG rats showed increased number of primordial follicles when compared with CTR animals, an observation corroborated by previous findings that hyperinsulinemia promotes follicle maturation arrest (Franks et al. 2000). Moreover, MSG rats exhibited a robust increase in follicular cysts and atretic follicle counts, suggesting an impaired ovarian microenvironment. Atretic primary follicles were visualized only in the ovaries of MSG rats (Supplementary Fig. 2), which denote a severe impairment on follicle development. Of note, all the abovementioned models that present PCO also exhibit hyperandrogenism; therefore, the fact that our MSG female rats exhibited PCO without hyperandrogenism reinforces the role of hyperinsulinemia as an inductor of PCO per se, without the contribution of overactivated HPG axis.

Nonetheless, another important contributor to ovarian follicle development is AMH. Our MSG rats had increased $\mathrm{AMH}$ immunostaining on antral follicles, even though it had not affected total AMH staining when compared with CTR rats. Previous reports showed that $\mathrm{AMH}$ functions as an inhibitory growth factor in the ovary during early stages of folliculogenesis (Durlinger et al. 2002) and increases primordial follicle population (Kevenaar et al. 2006), which corroborates our findings of primordial follicle pool and follicular atresia in MSG rats. Intriguingly, an increase in AMH production specifically by antral follicles was also observed in PCOS women (Bhide et al. 2015). Thus, these data suggest a synergic role for hyperinsulinemia and AMH overproduction in the pathogenesis of impaired follicle development along with augmented follicular atresia and cysts.

Taken together, our reproductive data support a PCOS-like phenotype in MSG obese female rats, with the presence of some key diagnostic criteria, namely oligoovulation and PCO. However, one should be aware that these rats do not mimic all physiological processes seen in human PCOS and other animal models, particularly because of the hypothalamic basilar nuclei damage of MSG model with consequent stunted growth, a feature not described in PCOS women. Additionally, our MSG rats presented unchanged serum reproductive hormones, a feature found in a minority of PCOS women, but that deserves further investigation. Therefore, one should be cautious when translating data from our MSG female rats to humans.
In conclusion, our MSG obese female rats reiterate the fundamental role of hyperinsulinemia, obesity, and adipocyte dysfunction in reproductive disorders. To the best of our knowledge, there is no other rodent model presenting the characteristics described herein, which opens up the perspective of investigating in vivo how MetS affects reproductive biology without the interference of exacerbated HPG axis function.

\section{Supplementary data}

This is linked to the online version of the paper at http://dx.doi.org/10.1530/ JOE-15-0453.

\section{Declaration of interest}

The authors declare that the research was conducted with no commercial or financial relationship that could be interpreted as a potential conflict of interest.

\section{Funding}

This study was funded by Fundação de Amparo à Pesquisa e ao Desenvolvimento Científico e Tecnológico do Maranhão-FAPEMA (Grant no. 00280/12 and scholarships to R S G, R O B, and J L L F) and Coordenação de Aperfeiçoamento de Pessoal de Nível Superior-CAPES (scholarship to C C V).

\section{Acknowledgments}

The authors are very grateful to $\mathrm{Dr}$ Deborah M Sloboda from the Department of Biochemistry and Biomedical Sciences, McMaster University, and Dr Farida Sohrabji from the Department of Neuroscience and Experimental Therapeutics, College of Medicine, Texas A\&M University, for critical discussion of the data. The authors would also like to thank the superb technical support given by Tiago da Silva Teófilo from the Laboratory of Histology, Federal University of Maranhão, and Marco Antônio Sales dos Santos and Laboratório Gaspar for the support given during the hormone assays.

\section{References}

Alberti KG, Eckel RH, Grundy SM, Zimmet PZ, Cleeman JI, Donato KA, Fruchart JC, James WP, Loria CM, Smith SC Jr, et al. 2009 Harmonizing the metabolic syndrome: a joint interim statement of the International Diabetes Federation Task Force on Epidemiology and Prevention; National Heart, Lung, and Blood Institute; American Heart Association; World Heart Federation; International Atherosclerosis Society; and International Association for the Study of Obesity. Circulation 120 1640-1645. (doi:10.1161/ CIRCULATIONAHA.109.192644)

Azziz R, Ehrmann D, Legro RS, Whitcomb RW, Hanley R, Fereshetian AG, O'Keefe M, Ghazzi MN \& the PCTS Group 2001 Troglitazone improves ovulation and hirsutism in the polycystic ovary syndrome: a multicenter, double blind, placebo-controlled trial. Journal of Clinical Endocrinology and Metabolism 86 1626-1632.

Published by Bioscientifica Ltd. 
Bernardis LL \& Patterson BD 1968 Correlation between 'Lee index' and carcass fat content in weanling and adult female rats with hypothalamic lesions. Journal of Endocrinology 40 527-528. (doi:10.1677/joe.0.0400527)

Bernuci MP, Szawka RE, Helena CV, Leite CM, Lara HE \& Anselmo-Franci JA 2008 Locus coeruleus mediates cold stress-induced polycystic ovary in rats. Endocrinology 149 2907-2916. (doi:10.1210/en.2007-1254)

Bhide P, Dilgil M, Gudi A, Shah A, Akwaa C \& Homburg R 2015 Each small antral follicle in ovaries of women with polycystic ovary syndrome produces more antimullerian hormone than its counterpart in a normal ovary: an observational cross-sectional study. Fertility and Sterility 103 537-541. (doi:10.1016/j.fertnstert.2014.10.033)

Bojanic V, Bojanic Z, Najman S, Savic T, Jakovljevic V, Najman S \& Jancic S 2009 Diltiazem prevention of toxic effects of monosodium glutamate on ovaries in rats. General Physiology and Biophysics $\mathbf{2 8}$ 149-154.

Brawer JR, Munoz M \& Farookhi R 1986 Development of the polycystic ovarian condition (PCO) in the estradiol valerate-treated rat. Biology of Reproduction 35 647-655. (doi:10.1095/biolreprod35.3.647)

Campos KE, Volpato GT, Calderon IM, Rudge MV \& Damasceno DC 2008 Effect of obesity on rat reproduction and on the development of their adult offspring. Brazilian Journal of Medical and Biological Research 41 122-125. (doi:10.1590/S0100-879X2008005000001)

Clemens JA, Roush ME, Fuller RW \& Shaar CJ 1978 Changes in luteinizing hormone and prolactin control mechanisms produced by glutamate lesions of the arcuate nucleus. Endocrinology 103 1304-1312. (doi:10.1210/endo-103-4-1304)

Connor KL, Vickers MH, Beltrand J, Meaney MJ \& Sloboda DM 2012 Nature, nurture or nutrition? Impact of maternal nutrition on maternal care, offspring development and reproductive function. Journal of Physiology 590 2167-2180. (doi:10.1113/ jphysiol.2011.223305)

Couillard C, Mauriege P, Imbeault P, Prud'homme D, Nadeau A, Tremblay A, Bouchard C \& Despres JP 2000 Hyperleptinemia is more closely associated with adipose cell hypertrophy than with adipose tissue hyperplasia. International Journal of Obesity and Related Metabolic Disorders 24 782-788. (doi:10.1038/ sj.ijo.0801227)

Creanga AA, Bradley HM, McCormick C \& Witkop CT 2008 Use of metformin in polycystic ovary syndrome: a meta-analysis. Obstetrics and Gynecology 111 959-968.

Dada MO \& Blake CA 1985 Monosodium L-glutamate administration: effects on gonadotrophin secretion, gonadotrophs and mammotrophs in prepubertal female rats. Journal of Endocrinology 104 185-192. (doi:10.1677/joe.0.1040185)

Das RS \& Ghosh SK 2011 Long-term effects in ovaries of the adult mice following exposure to monosodium glutamate during neonatal life-a histological study. Nepal Medical College Journal 13 77-83.

Dumesic DA, Oberfield SE, Stener-Victorin E, Marshall JC, Laven JS \& Legro RS 2015 Scientific statement on the diagnostic criteria, epidemiology, pathophysiology, and molecular genetics of polycystic ovary syndrome. Endocrine Reviews 36 487-525. (doi:10.1210/ er.2015-1018)

Durlinger AL, Gruijters MJ, Kramer P, Karels B, Ingraham HA, Nachtigal MW, Uilenbroek JT, Grootegoed JA \& Themmen AP 2002 Anti-Mullerian hormone inhibits initiation of primordial follicle growth in the mouse ovary. Endocrinology 143 1076-1084. (doi:10.1210/endo.143.3.8691)

Duval K, Langlois MF, Carranza-Mamane B, Pesant MH, Hivert MF, Poder TG, Lavoie HB, Ainmelk Y, St-Cyr Tribble D, Laredo S, et al. 2015 The Obesity-Fertility Protocol: a randomized controlled trial assessing clinical outcomes and costs of a transferable interdisciplinary lifestyle intervention, before and during pregnancy, in obese infertile women. BMC Obesity 2 47. (doi:10.1186/s40608015-0077-x)
Escobar-Morreale HF \& San Millan JL 2007 Abdominal adiposity and the polycystic ovary syndrome. Trends in Endocrinology \& Metabolism 18 266-272. (doi:10.1016/j.tem.2007.07.003)

Franca LR, Suescun MO, Miranda JR, Giovambattista A, Perello M, Spinedi E \& Calandra RS 2006 Testis structure and function in a nongenetic hyperadipose rat model at prepubertal and adult ages. Endocrinology 147 1556-1563. (doi:10.1210/en.2005-0640)

Franca LM, Freitas LN, Chagas VT, Coelho CF, Barroso WA, Costa GC, Silva LA, Debbas V, Laurindo FR \& Paes AM 2014 Mechanisms underlying hypertriglyceridemia in rats with monosodium L-glutamate-induced obesity: evidence of XBP-1/PDI/MTP axis activation. Biochemical and Biophysical Research Communications 443 725-730. (doi:10.1016/j.bbrc.2013.12.042)

Franks S, Mason H \& Willis D 2000 Follicular dynamics in the polycystic ovary syndrome. Molecular and Cellular Endocrinology 163 49-52. (doi:10.1016/S0303-7207(99)00239-7)

Gao M, Ma Y \& Liu D 2015 High-fat diet-induced adiposity, adipose inflammation, hepatic steatosis and hyperinsulinemia in outbred CD-1 mice. PLOS ONE 10 e0119784.

Hirshfield AN \& Midgley AR Jr 1978 Morphometric analysis of follicular development in the rat. Biology of Reproduction 19 597-605. (doi:10.1095/biolreprod19.3.597)

Kevenaar ME, Meerasahib MF, Kramer P, van de Lang-Born BM, de Jong FH, Groome NP, Themmen AP \& Visser JA 2006 Serum anti-mullerian hormone levels reflect the size of the primordial follicle pool in mice. Endocrinology 147 3228-3234. (doi:10.1210/ en.2005-1588)

Knudsen KL, Blank SK, Burt Solorzano C, Patrie JT, Chang RJ, Caprio S, Marshall JC \& McCartney CR 2010 Hyperandrogenemia in obese peripubertal girls: correlates and potential etiological determinants Obesity 18 2118-2124.

Lamperti A \& Blaha G 1976 The effects of neonatally-administered monosodium glutamate on the reproductive system of adult hamsters. Biology of Reproduction 14 362-369. (doi:10.1095/ biolreprod14.3.362)

Lamperti AA \& Baldwin DM 1983 Effects of anterior hypothalamic deafferentation and arcuate nucleus lesions on the feedback actions of estradiol benzoate on luteinizing hormone and follicle-stimulating hormone secretion in the female hamster. Biology of Reproduction $\mathbf{2 8}$ 745-751. (doi:10.1095/biolreprod28.3.745)

Lara HE, Dissen GA, Leyton V, Paredes A, Fuenzalida H, Fiedler JL \& Ojeda SR 2000 An increased intraovarian synthesis of nerve growth factor and its low affinity receptor is a principal component of steroid-induced polycystic ovary in the rat. Endocrinology 141 1059-1072. (doi:10.1210/endo.141.3.7395)

Liu SN, Liu Q, Li LY, Huan Y, Sun SJ \& Shen ZF 2011 Long-term fenofibrate treatment impaired glucose-stimulated insulin secretion and up-regulated pancreatic NF-kappa B and iNOS expression in monosodium glutamate-induced obese rats: is that a latent disadvantage? Journal of Translational Medicine 9176. (doi:10.1186/1479-5876-9-176)

Lorden JF \& Caudle A 1986 Behavioral and endocrinological effects of single injections of monosodium glutamate in the mouse. Neurobehavioral Toxicology and Teratology 8 509-519.

Maiter D, Underwood LE, Martin JB \& Koenig JI 1991 Neonatal treatment with monosodium glutamate: effects of prolonged growth hormone (GH)-releasing hormone deficiency on pulsatile GH secretion and growth in female rats. Endocrinology 128 1100-1106. (doi:10.1210/ endo-128-2-1100)

Manneras L, Cajander S, Holmang A, Seleskovic Z, Lystig T, Lonn M \& Stener-Victorin E 2007 A new rat model exhibiting both ovarian and metabolic characteristics of polycystic ovary syndrome. Endocrinology 148 3781-3791. (doi:10.1210/en.2007-0168)

Manneras-Holm L, Leonhardt H, Kullberg J, Jennische E, Oden A, Holm G, Hellstrom M, Lonn L, Olivecrona G, Stener-Victorin E, et al. 2011 Adipose tissue has aberrant morphology and function in PCOS:

Published by Bioscientifica Ltd 
enlarged adipocytes and low serum adiponectin, but not circulating sex steroids, are strongly associated with insulin resistance. Journal of Clinical Endocrinology \& Metabolism 96 E304-E311. (doi:10.1210/ jc.2010-1290)

Marcondes FK, Bianchi FJ \& Tanno AP 2002 Determination of the estrous cycle phases of rats: some helpful considerations. Brazilian Journal of Biology 62 609-614. (doi:10.1590/S1519-69842002000400008)

Miranda RA, Agostinho AR, Trevenzoli IH, Barella LF, Franco CC, Trombini AB, Malta A, Gravena C, Torrezan R, Mathias PC, et al. 2014 Insulin oversecretion in MSG-obese rats is related to alterations in cholinergic muscarinic receptor subtypes in pancreatic islets. Cellular Physiology and Biochemistry 33 1075-1086. (doi:10.1159/000358677)

Moreno G, Perello M, Camihort G, Luna G, Console G, Gaillard RC \& Spinedi E 2006 Impact of transient correction of increased adrenocortical activity in hypothalamo-damaged, hyperadipose female rats. International Journal of Obesity 30 73-82. (doi:10.1038/ sj.ijo.0803109)

Nagasawa H, Yanai R \& Kikuyama S 1974 Irreversible inhibition of pituitary prolactin and growth hormone secretion and of mammary gland development in mice by monosodium glutamate administered neonatally. Acta Endocrinologica 75 249-259.

Nemeroff CB, Lamartiniere CA, Mason GA, Squibb RE, Hong JS \& Bondy SC 1981 Marked reduction in gonadal steroid hormone levels in rats treated neonatally with monosodium L-glutamate: further evidence for disruption of hypothalamic-pituitary-gonadal axis regulation. Neuroendocrinology 33 265-267. (doi:10.1159/000123243)

Newell-Fugate AE, Taibl JN, Clark SG, Alloosh M, Sturek M \& Krisher RL 2014 Effects of diet-induced obesity on metabolic parameters and reproductive function in female Ossabaw minipigs. Comparative Medicine 64 44-49.

Norman RJ, Davies MJ, Lord J \& Moran LJ 2002 The role of lifestyle modification in polycystic ovary syndrome. Trends in Endocrinology and Metabolism 13 251-257.

O’Neill S \& O'Driscoll L 2015 Metabolic syndrome: a closer look at the growing epidemic and its associated pathologies. Obesity Reviews 16 1-12. (doi:10.1111/obr.12229)

Olney JW 1969 Brain lesions, obesity, and other disturbances in mice treated with monosodium glutamate. Science 164 719-721. (doi:10.1126/science.164.3880.719)

Olney JW \& Sharpe LG 1969 Brain lesions in an infant rhesus monkey treated with monsodium glutamate. Science 166 386-388. (doi:10.1126/science.166.3903.386)

Radavelli-Bagatini S, Blair AR, Proietto J, Spritzer PM \& Andrikopoulos S 2011 The New Zealand obese mouse model of obesity insulin resistance and poor breeding performance: evaluation of ovarian structure and function. Journal of Endocrinology 209 307-315. (doi:10.1530/JOE-11-0022)

Ribeiro RA, Santos-Silva JC, Vettorazzi JF, Cotrim BB, Boschero AC \& Carneiro EM 2015 Taurine supplementation enhances insulin secretion without altering islet morphology in non-obese diabetic mice. Advances in Experimental Medicine and Biology 803 353-370. (doi:10.1007/978-3-319-15126-7)

Rose PA \& Weick RF 1987 Evidence for reorganization of the neuroendocrine centres regulating pulsatile $\mathrm{LH}$ secretion in rats receiving neonatal monosodium-L-glutamate treatment. Journal of Endocrinology 113 261-269. (doi:10.1677/joe.0.1130261)

Rotterdam EA-SPCWG 2004 Revised 2003 consensus on diagnostic criteria and long-term health risks related to polycystic ovary syndrome. Fertility and Sterility 81 19-25. (doi:10.1016/j. fertnstert.2003.10.004)
Sanchez-Garrido MA, Ruiz-Pino F, Manfredi-Lozano M, Leon S, Heras V, Castellano JM, Castano JP, Luque RM, Vazquez MJ, Roa J, et al. 2015 Metabolic and gonadotropic impact of sequential obesogenic insults in the female: influence of the loss of ovarian secretion. Endocrinology 156 2984-2998. (doi:10.1210/en.2014-1951)

Sasaki F \& Sano M 1986 Roles of the arcuate nucleus and ovary in the maturation of growth hormone, prolactin, and nongranulated cells in the mouse adenohypophysis during postnatal development: a stereological morphometric study by electron microscopy. Endocrinology 119 1682-1689. (doi:10.1210/endo-119-4-1682)

Shi D, Dyck MK, Uwiera RR, Russell JC, Proctor SD \& Vine DF 2009 A unique rodent model of cardiometabolic risk associated with the metabolic syndrome and polycystic ovary syndrome. Endocrinology 150 4425-4436. (doi:10.1210/en.2008-1612)

Simental-Mendia LE, Rodriguez-Moran M \& Guerrero-Romero F 2008 The product of fasting glucose and triglycerides as surrogate for identifying insulin resistance in apparently healthy subjects. Metabolic Syndrome and Related Disorders 6 299-304. (doi:10.1089/ met.2008.0034)

Tosi F, Negri C, Perrone F, Dorizzi R, Castello R, Bonora E \& Moghetti P 2012 Hyperinsulinemia amplifies GnRH agonist stimulated ovarian steroid secretion in women with polycystic ovary syndrome. Journal of Clinical Endocrinology and Metabolism 97 1712-1719.

Vryonidou A, Paschou SA, Muscogiuri G, Orio F \& Goulis DG 2015 MECHANISMS IN ENDOCRINOLOGY: Metabolic syndrome through the female life cycle. European Journal of Endocrinology 173 R153-R163.

Wallace TM, Levy JC \& Matthews DR 2004 Use and abuse of HOMA modeling. Diabetes Care 27 1487-1495. (doi:10.2337/ diacare.27.6.1487)

Walters KA, Allan CM \& Handelsman DJ 2012 Rodent models for human polycystic ovary syndrome. Biology of Reproduction 86 149, 141-112.

Watanobe H 2002 Leptin directly acts within the hypothalamus to stimulate gonadotropin-releasing hormone secretion in vivo in rats. Journal of Physiology 545 255-268. (doi:10.1113/ jphysiol.2002.023895)

Webber LJ, Stubbs S, Stark J, Trew GH, Margara R, Hardy K \& Franks S 2003 Formation and early development of follicles in the polycystic ovary. Lancet 362 1017-1021. (doi:10.1016/ S0140-6736(03)14410-8)

Wells JC, Marphatia AA, Cole TJ \& McCoy D 2012 Associations of economic and gender inequality with global obesity prevalence: understanding the female excess. Social Science \& Medicine $\mathbf{7 5}$ 482-490.

Wright CS, Hovatta O, Margara R, Trew G, Winston RM, Franks S \& Hardy K 1999 Effects of follicle-stimulating hormone and serum substitution on the in-vitro growth of human ovarian follicles. Human Reproduction 14 1555-1562. (doi:10.1093/ humrep/14.6.1555)

Wu C, Lin F, Qiu S \& Jiang Z 2014a The characterization of obese polycystic ovary syndrome rat model suitable for exercise intervention. PLOS ONE 9 e99155. (doi:10.1371/journal. pone.0099155)

Wu S, Divall S, Nwaopara A, Radovick S, Wondisford F, Ko C \& Wolfe A $2014 b$ Obesity-induced infertility and hyperandrogenism are corrected by deletion of the insulin receptor in the ovarian theca cell. Diabetes 63 1270-1282. (doi:10.2337/db13-1514)

Zhang N, Huan Y, Huang H, Song GM, Sun SJ \& Shen ZF 2010 Atorvastatin improves insulin sensitivity in mice with obesity induced by monosodium glutamate. Acta Pharmaceutica Sinica $\mathbf{3 1}$ 35-42. (doi:10.1038/aps.2009.176)

Received in final form 1 March 2016

Accepted 7 March 2016

Accepted Preprint published online 7 March 2016 http://joe.endocrinology-journals.org

DOI: $10.1530 / \mathrm{JOE}-15-0453$
(C) 2016 Society for Endocrinology Printed in Great Britain
Published by Bioscientifica Ltd. 